Gefässchirurgie $2015 \cdot 20: 304-312$

DOI 10.1007/s00772-015-0048-z

Online publiziert: 26. Juni 2015

c) Springer-Verlag Berlin Heidelberg 2015

\section{H.-H. Eckstein · H. Söllner · A. Kühnl}

Klinik und Poliklinik für Vaskuläre und Endovaskuläre Chirurgie, Klinikum rechts

der Isar, Technische Universität München, München, Deutschland

\title{
Die Zeitschrift Gefässchirurgie von 1996 bis 2015 - Bibliometrische Analyse und inhaltliche Perspektiven
}

Seit August 2014 wird eine z. T. kontroverse Diskussion um die weitere Entwicklung der Zeitschrift Gefässchirurgie geführt. Strittige Punkte sind a) die Sinnhaftigkeit bzw. die Notwendigkeit einer Listung der Gefässchirurgie beim Institute for Scientific Information (ISI) inkl. des damit verbundenen Journal Impact Faktors (JIF bzw. IF) sowie b) die Frage nach einer fachübergreifenden, internationalen Sichtbarkeit der Gefässchirurgie. Seitens der Schriftleitung wurde im Editorial des Hefts 7/2014 ein mit dem Editorial Board abgestimmter Vorschlag vorgestellt [1]. Dieser sah die Schaffung eines sog. Hybrid-Journals vor, bestehend aus einer weitestgehend unveränderten deutschsprachigen Printversion sowie einer Open-access-Verfügbarkeit von guten, ins Englische übersetzten Artikeln. Diese qualitativ hochwertigen Arbeiten sollten dann in PubMed Central (PMC) jederzeit, weltweit und kostenfrei abrufbar sein. Dieser Vorschlag hat drei wesentliche Vorteile:

1. Autoren haben die Chance weltweit wahrgenommen zu werden, obgleich Gefässchirurgie (noch) nicht in MEDLINE gelistet ist.

2. Möglichst viele Zitationen dieser PMC-Artikel erhöhen die Chancen einer Listung beim ISI und eines erneuten JIF.

3. Das Niveau der Gefässchirurgie wird weiter verbessert.

Nach diesem Muster agieren z. B. das Deutsche Ärzteblatt $[2,3]$ und andere Journals wie z. B das Journal der Deutschen Dermatologischen Gesellschaft [4] und das British Journal of Psychiatric Bul- letin (Organ des Royal College of Psychiatrists, [5]) sehr erfolgreich. Die Schriftleitung hatte darüber hinaus eine Umbenennung in Gefässchirurgie International vorgeschlagen (entlehnt vom Deutschen Ärzteblatt International). Dies hätte unseren Anspruch, fachübergreifend und außerhalb des deutschsprachigen Bereichs besser wahrgenommen zu werden, sehr klar zum Ausdruck gebracht.

$\mathrm{Zu}$ keinem Zeitpunkt sollte neben der deutschsprachigen Gefässchirurgie ein separates, englischsprachiges Online-Journal auf den Weg gebracht werden, da diese - nur auf den ersten Blick attraktive Lösung (einfache Wege zur Veröffentlichung, Verbleib der Rechte bei den Autoren etc.) - auch Nachteile gehabt hätte. So hätte u. a. ein neues Editorial Board und eine völlig neue Homepage eingerichtet werden müssen. Die Trennung von dem Printjournal Gefässchirurgie hätte außerdem bedeutet, dass die Artikel dieses neuen gefäßchirurgischen Online-Journals für die Mitglieder der drei Fachgesellschaften (Deutsche Gesellschaft für Ge- fäßchirurgie und Gefäßmedizin/DGG, Schweizerische Gesellschaft für Gefäßchirurgie/SGG, Österreichische Gesellschaft für Gefäßchirurgie/ÖGG) nur online sichtbar gewesen wären; ein gravierender Nachteil für Autoren und Leser, da reine Online-Journals nur durch einen direkten Aufruf wahrgenommen werden können. Folgerichtig hatte das Editorial Board von Gefässchirurgie diese Option bereits im September 2014 abgelehnt.

Schon bisher ist es möglich, gegen eine Gebühr ("article processing charges" bzw. APC, z. B. 3.000 US-Dollar bei Elsevier, $2.200 €$ bei Springer) eigene Print-Artikel online frei zugänglich zu machen; so auch bei Gefässchirurgie. Diese Kosten müssen aber die Autoren bzw. deren Institutionen selbst tragen. Der Plan für Gefässchirurgie sah vor, diese Kosten - zumindest für eine gewisse Zeit - seitens der drei Fachgesellschaften zu übernehmen. Je nach Anzahl von PMC-Artikeln hätten sich die Kosten hierfür in einem Bereich von $20.000 €(1$ PMC Artikel/Heft) bis maximal $50.000 €$ (2-3 PMC Artikel/Heft) bewegt. Mit einer

Tab. 1 Strukturelle Entwicklung der Zeitschrift Gefässchirurgie und Anzahl der jährlichen Dokumente seit 1996 (Quelle: SCOPUS, [6])

\begin{tabular}{|c|c|c|}
\hline Meilensteine & Jahr & \multirow{8}{*}{ 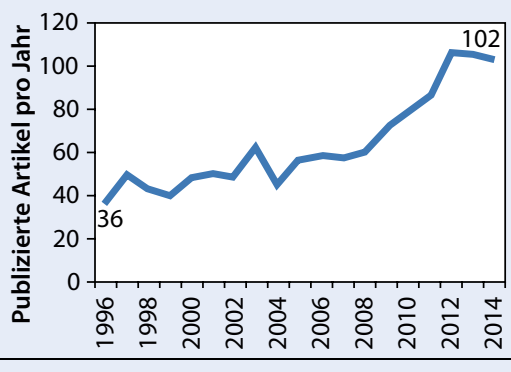 } \\
\hline Erste Publikation mit 4 Heften/Jahr) & 1996 & \\
\hline Online-Archiv Springer Link & seit 1997 & \\
\hline Vierfarbiger Druck (zuvor zweifarbig) & seit 2005 & \\
\hline CME-Arti & seit 2005 & \\
\hline Verdoppelung der Heftanzahl auf 8/Jahr & seit 2009 & \\
\hline Editorial Manager & seit 2009 & \\
\hline Erster Impact Factor 2009 & 2009 & \\
\hline
\end{tabular}



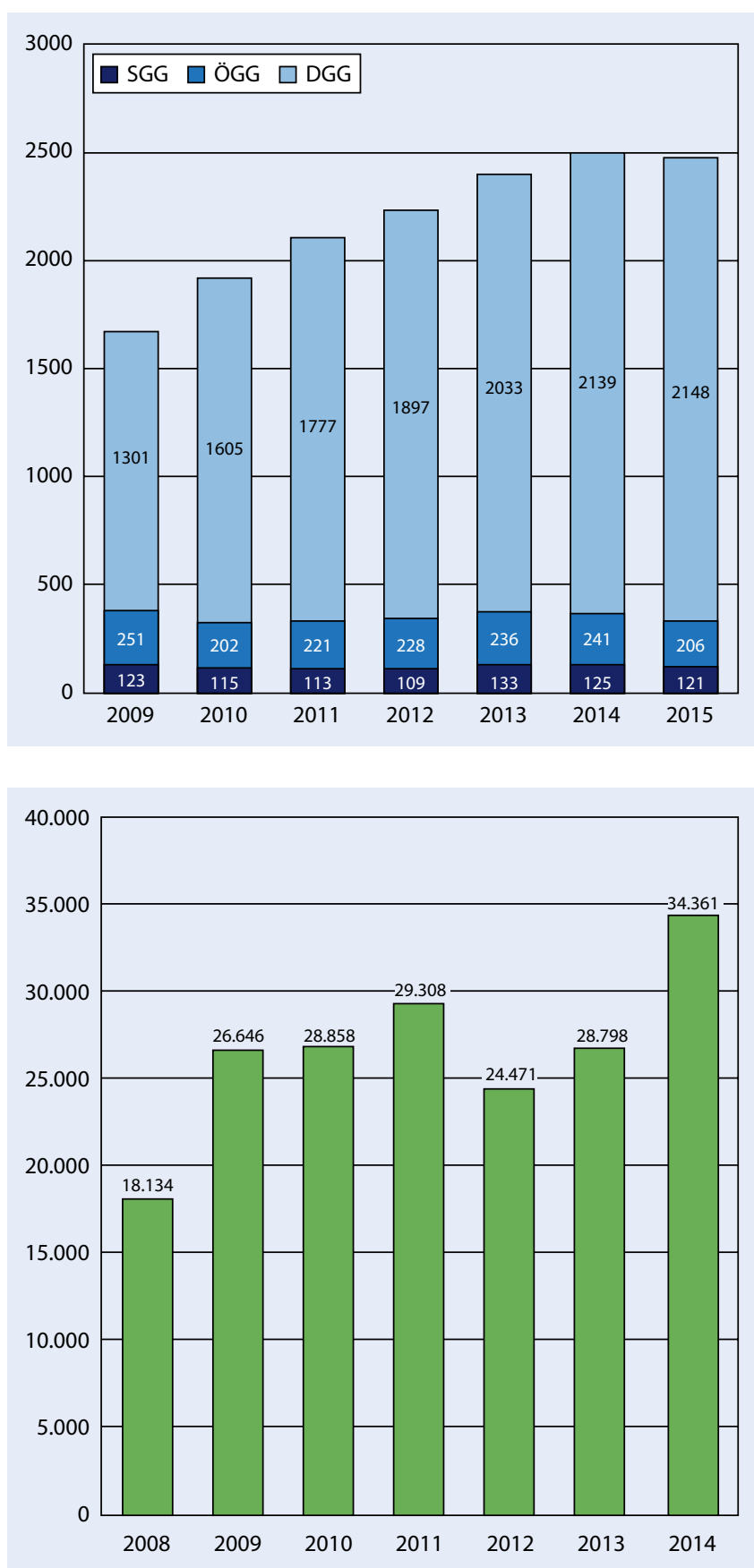

Abb. $1<$ Abonnemententwicklung der Zeitschrift Gefässchirurgie durch Mitglieder der Deutschen Gesellschaft für Gefäßchirurgie und Gefäßmedizin (DGG), der Österreichischen Gesellschaft für Gefäßchirurgie (ÖGG) und der Schweizerischen Gesellschaft für Gefäßchirurgie (SGG) seit 2009. Freie Abonnenten nicht enthalten (Quelle: Springer Verlag)

Abb. $2 \triangleleft$ Nutzung des Online-Archivs von Gefässchirurgie - Volltextzugriffe 2008 bis 2014 (Quelle: Springer Verlag)

moderaten Erhöhung der Mitgliedsbeiträge um 20-25 €/Jahr hätte die DGG bei mittlerweile $>2,000$ Mitgliedern die notwendigen Summen relativ leicht generieren können. Während die SGG sich mit diesen Gedanken rasch anfreunden konnte, erfolgte seitens der ÖGG und der DGG im Herbst 2014 eine klare Ablehnung. Zum Zeitpunkt der Abfassung zeichnet sich bei der DGG ein vorsichtiges Umdenken ab (s. Editorial und Artikel von Prof. Debus et al. in diesem Heft).
NE und PMC zu den Stichworten medical journal und impact factor.

\section{Strukturelle und bibliometrische Analyse der Zeitschrift Gefässchirurgie}

Die Zeitschrift Gefässchirurgie erschien in Nachfolge der Zeitschrift Angio erstmals im Jahr 1996 (Schriftleitung J.R. Allenberg). Die strukturelle Weiterentwicklung ist der $\bullet$ Tab. $1 \mathrm{zu}$ entnehmen. Seit 2009 erscheint die Gefässchirurgie 8-mal pro Jahr. Parallel hierzu wurde die Anzahl der jährlich publizierten Dokumente über die Jahre deutlich gesteigert. Die Zunahme der Mitgliederzahl, insbesondere der DGG, hat zu einem ständigen Anstieg der Abonnentenzahl geführt. Aktuell finden sich hierunter 2148 Mitglieder der DGG, 206 ÖGG- und 121 SGG-Mitglieder. Hinzu kommen weitere freie Abonnenten (• Abb. 1). Darüber hinaus hat die Nutzung des Online-Archivs für Volltextzugriffe in den letzten Jahren ständig zugenommen (• Abb. 2).

\section{Zitationen von Publikationen aus der Zeitschrift Gefässchirurgie}

- Tab 2 und 3 zeigen die am meisten zitierten Publikationen aus Gefässchirurgie. Bei Betrachtung des Gesamtzeitraums seit 1996, zeigt sich, dass bis auf zwei Ausnahmen Publikationen aus den 1990er Jahren am häufigsten zitiert worden sind (- Tab. 2). Da bei allen bibliometrischen Rankings medizinischer Zeitschriften die Häufigkeit von Zitationen der letzten Jahre veranschlagt wird, wurde für die vorliegende Analyse die zwischen 2011 und 2014 erschienenen Arbeiten gezählt. Hierbei zeigt sich, dass insbesondere die S3-Leitlinie zur Karotisstenosehäufig zitiert worden ist (4-mal unter den 10 am häufigsten zitierten Arbeiten, - Tab. 3). Insgesamt werden Übersichts- und Review-Artikel, Stellungnahmen der Fachgesellschaften, Leitlinien und Publikationen aus Qualitätssicherungsprojekten am häufigsten zitiert. Wissenschaftliche Originalarbeiten finden sich nicht unter den jeweiligen „Top-10-Publikationen“. 


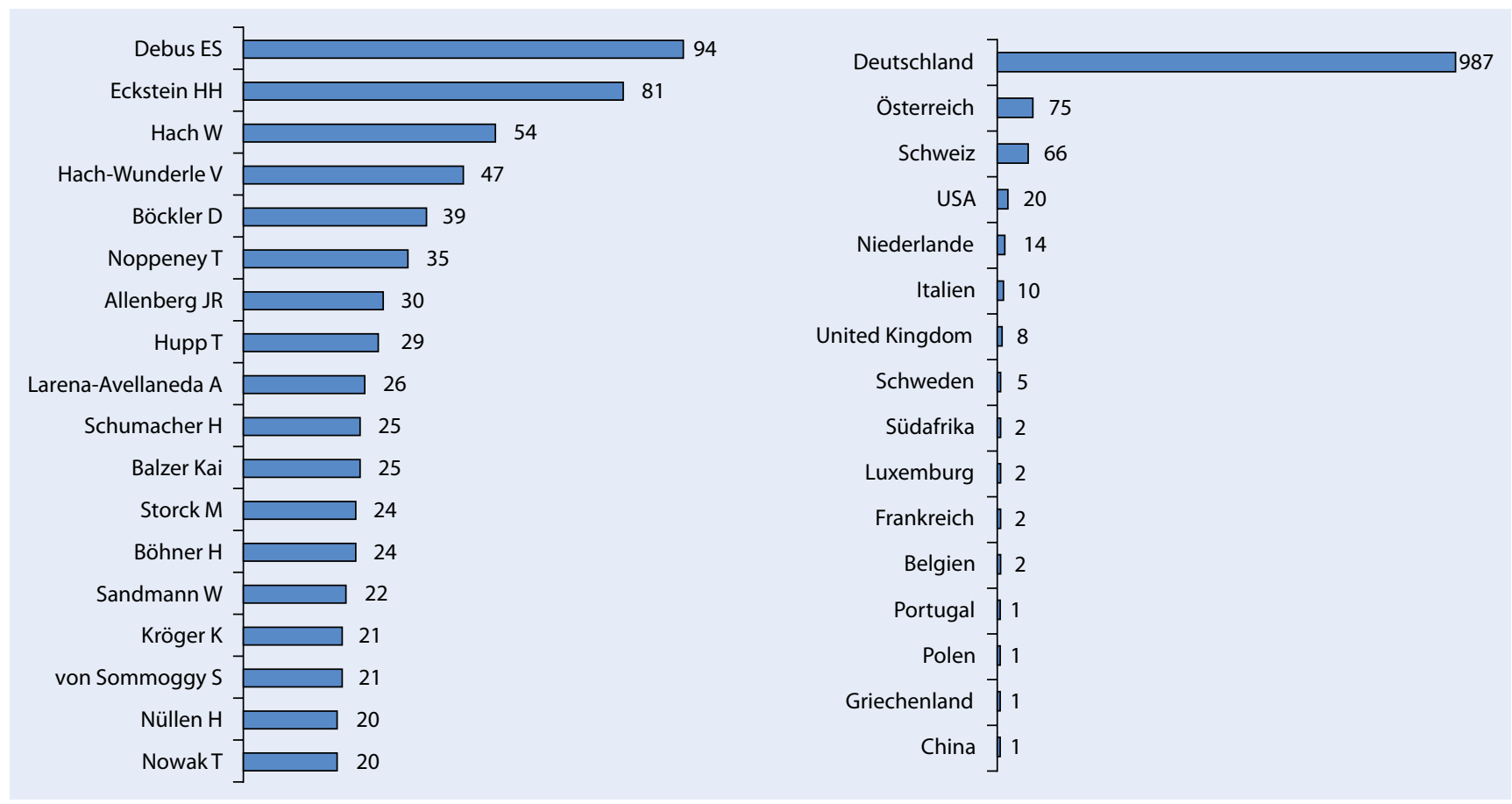

Abb. $3 \Delta$ Autoren mit mindestens 20 Publikationen in Gefässchirurgie und Herkunftsländer aller Publikationen (1996- Mai 2015, Quelle: SCOPUS, [6])

\section{Autoren und Herkunftsländer der Publikationen in Gefässchirurgie}

- Abb. 3 zeigt die Autoren (Erst- und CoAutorenschaften) mit den meisten publizierten Dokumenten sowie die Herkunftsländer der Publikationen. Da Schriftleiter und Rubrikherausgeber „kraft Amtes“ aufgefordert sind, Inhalte beizusteuern, verwundert es nicht, dass relativ wenige Autoren viele Dokumente für die Gefässchirurgie verfasst haben. Zukünftig wäre es allerdings wünschenswert, die Anzahl von Autoren zu erhöhen.

Wie zu erwarten war, stammen die meisten Publikationen aus Deutschland, gefolgt von Österreich und der Schweiz. Insgesamt sind bisher wenige Publikationen aus dem nicht deutschsprachigen Ausland erschienen. Am häufigsten sind hier die USA, die Niederlande, Italien, Großbritannien und Schweden vertreten (• Abb. 3).

\section{Gefässchirurgie im Vergleich mit nationalen und internationalen gefäßchirurgischen/ gefäßmedizinischen Journalen}

Das Portal SCImago bietet neben detaillierten Analysen zu einzelnen Zeitschriften auch die Möglichkeit eines unmittelbaren Vergleichs verschiedener Zeitschriften, Regionen und Ländern. Darüber hinaus werden für verschiedene Bereiche innerhalb der Medizin jährlich aktualisierte Ranglisten erstellt, die auf der Datenbank SCOPUS basieren. $\bullet$ Tab. 4 zeigt, das aktuelle Ranking (2013) der Gefässchirurgie bei SCImago für den Bereich „Cardiology and Cardiovascular Medicine" und „Surgery" (jeweils international und in Deutschland). Es zeigt sich, dass Gefässchirurgie auch bei diesen Rankings (welche nicht auf dem IF beruhen!) nur hintere Plätze einnimmt.

Im „Web of Knowledge“ des ISI erfolgt ein Ranking auf Basis der IF-Werte, mit allerdings deutlich weniger Journalen. Der mediane IF aller Zeitschriften dieser Rubrik lag im Jahre 2012 bei 2,399. Hier erreichte Gefässchirurgie (IF 0,235) im Jahr 2012 in der Rubrik „peripheral vascular disease“ Platz 63 von 68. Dies hat si- cherlich dazu beigetragen, dass Gefässchirurgie nicht länger beim ISI gelistet wird.

Die vergleichende SCImago-Analyse mit anderen relevanten gefäßchirurgischen/gefäßmedizinischen Zeitschriften bestätigt, dass aus der Gefässchirurgie mit 0,310 Zitationen pro publizierten Artikel über einen 3-Jahres-Zeitraum nur sehr selten zitiert wird. Dies betrifft sowohl den Vergleich mit internationalen „vaskulären Zeitschriften" (VASA, Eur J Vasc Endovasc Surg, Ann Vasc Surg, • Abb. 4a), den Vergleich mit deutschsprachigen chirurgischen Fachzeitschriften (Handchirurgie, Mikrochirurgie und Plastische Chirurgie, Operative Orthopädie und Traumatologie, - Abb. 4b) und den Vergleich mit deutschsprachigen allgemeinchirurgischen Zeitschriften (Der Chirurg, Zentralblatt für Chirurgie) und der Zeitschrift Phlebologie (• Abb. 4c). Einzig die Zeitschrift für Herz-, Thorax- und Gefäßchirurgie wird mit ca. 0,136 Zitationen/Artikel noch seltener zitiert (• Abb. 4b). Die Deutsche Gesellschaft für Herz-, Thorax und Gefäßchirurgie (DGHTG) verfügt jedoch mit dem Journal The Thoracic and Cardiovascular Surgeon (IF 1,075) über ein weiteres wissenschaftliches Organ. 


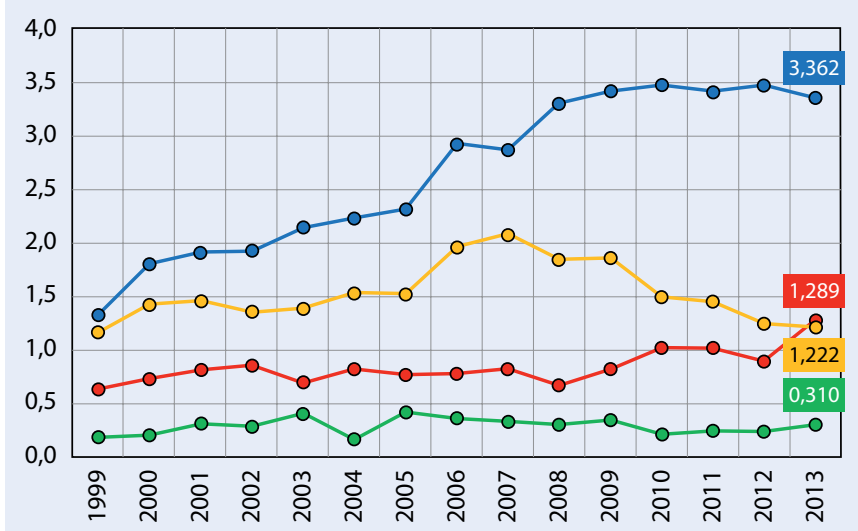

a
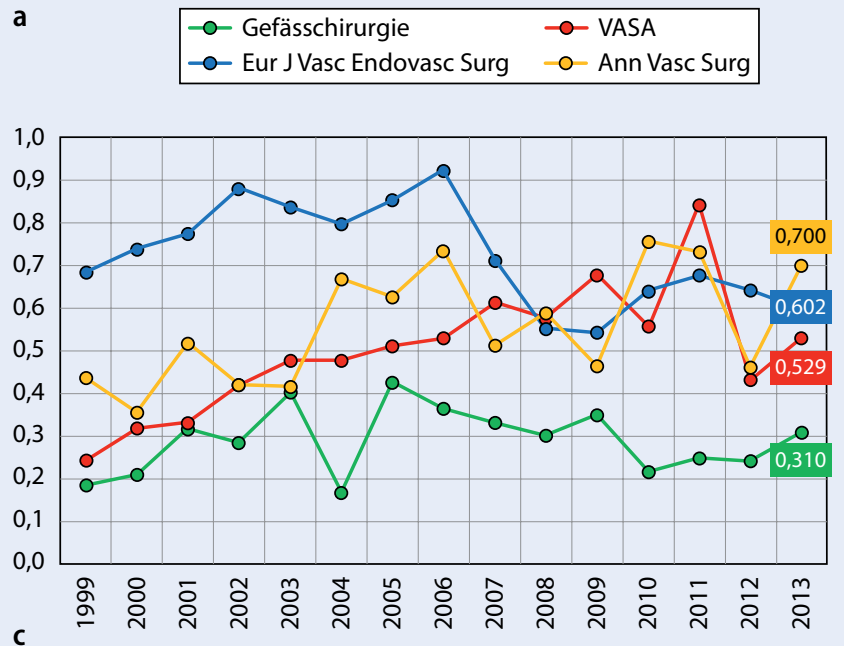

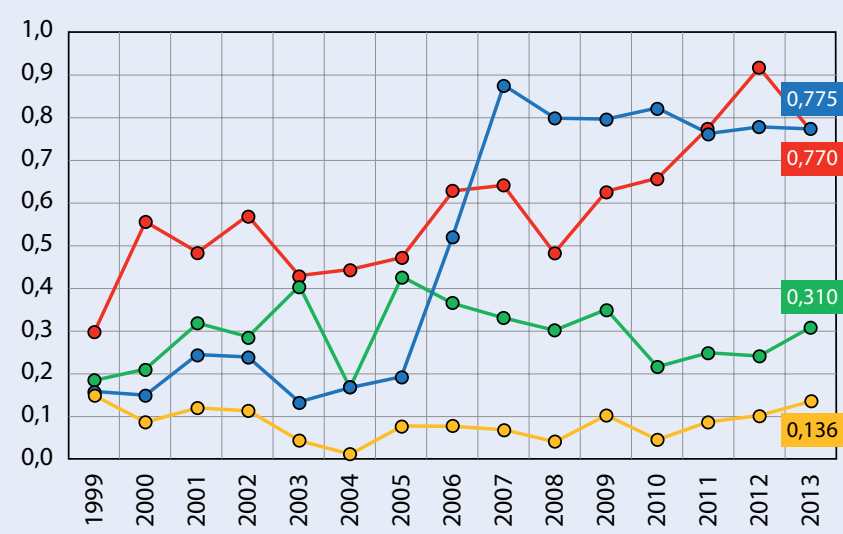

b
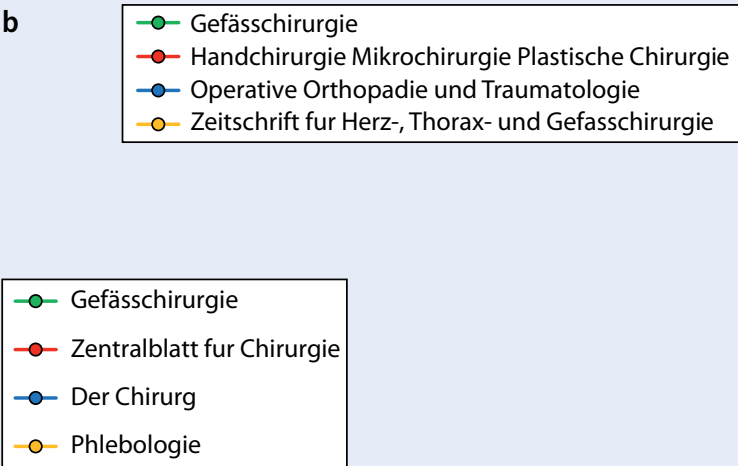

Abb. $4 \Delta$ a Zitationen pro Dokument (3-Jahres-Zeitraum) in der Gefässchirurgie und in weiteren internationalen gefäßchirurgischen/gefäßmedizinischen Journalen, b Zitationen pro Dokument (3-Jahres-Zeitraum) in deutschsprachigen chirurgischen Fachzeitschriften, c Zitationen pro Dokument (3-Jahres-Zeitraum) in deutschsprachigen allgemeinchirurgischen Zeitschriften sowie in der Zeitschrift Phlebologie (Quelle: SCImago) [7]

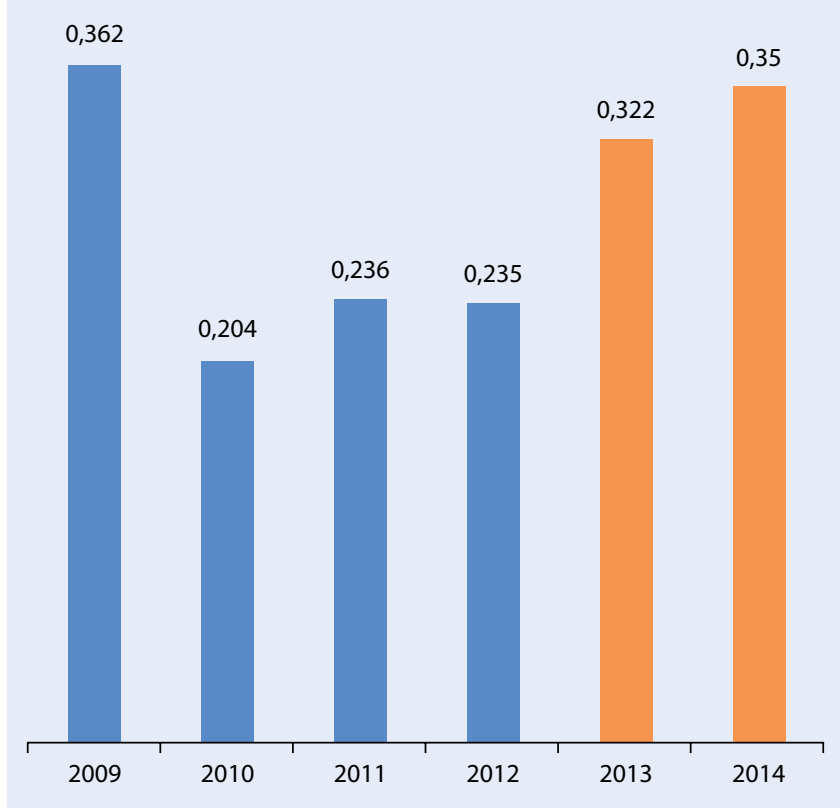

Abb. $5<$ Impact Factor von Gefässchirurgie seit 2009 (2013 und 2014 inoffiziell). (Quelle: Web of Science, SCOPUS [6])

\section{Impact Factor (IF) der Zeitschrift Gefässchirurgie}

Die Zeitschrift Gefässchirurgie hat im Jahre 2009 erstmals einen IF erhalten (basierend auf den Publikationen der Jahre 2007 und 2008). Die zuerkannten IFWerte sind $\bullet$ Abb. 5 zu entnehmen. Für 2013 und 2014 wurde der sog. ,,inoffizielle IF" anhand der in der Datenbank SCOPUS aufgeführten Zitationen von Publikationen aus Gefässchirurgie berechnet. Die Analyse von SCImago (die auf SCOPUS basiert) zeigt, dass die absolute Anzahl von Zitationen über die Jahre zugenommen hat (z. B. 62 Zitationen im Jahre 2013) Abb. 6. Allerdings ist die Anzahl der sog. Eigenzitationen mit ca. $35 \%$ (22/62) immer noch zu hoch. Hier sollte ein Anteil von maximal $25 \%$ Eigenzitationen nicht überschritten werden. 


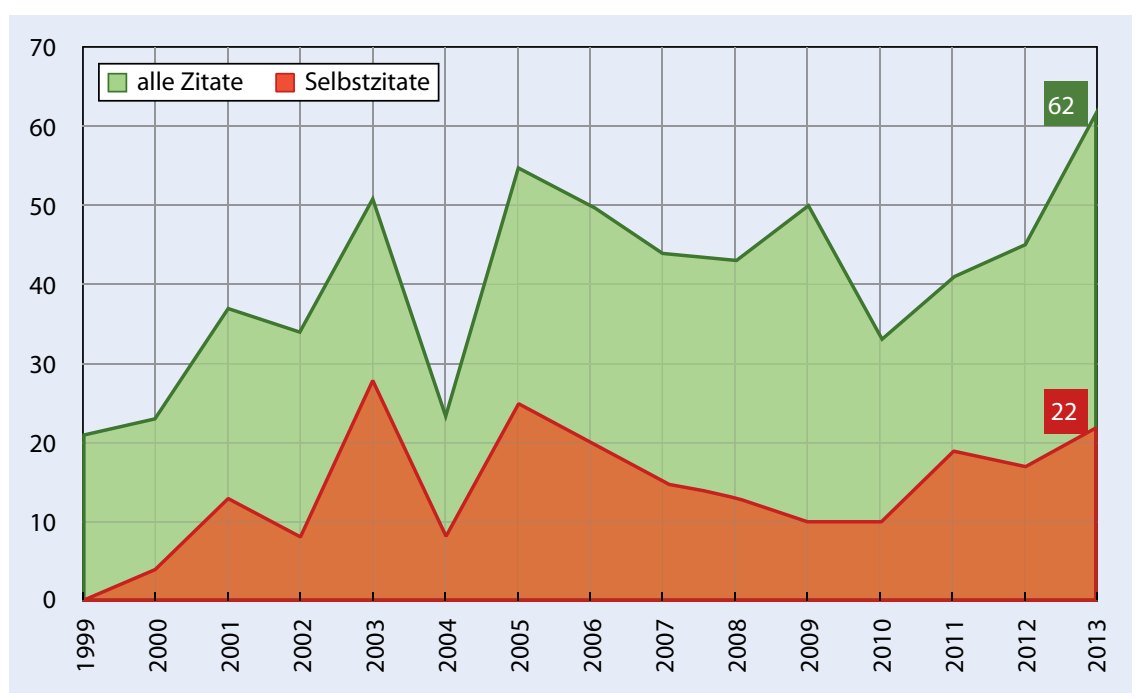

Abb. $6 \Delta$ Anzahl aller Zitationen (3-Jahres-Zeitraum) und sog. Eigenzitationen in Gefässchirurgie zwischen 1999 und 2013 (Quelle: SCIMAGO, [7])

\section{Der Journal Impact Factor (JIF): Rankinginstrument für (medizinische) Fachzeitschriften}

Der Journal Impact Factor (JIF bzw. IF) wurde 1972 von Eugene Garfield als Instrument für die Evaluation wissenschaftlicher Fachzeitschriften vorgestellt [9] und 1975 vom Institute for Scientific Information (ISI) übernommen [10]. Er wird jedes Jahr aus dem Verhältnis der Anzahl zitierter Artikel (Zähler) zur Anzahl aller zitierfähigen Artikel im vorausgegangenen 2-Jahres-Zeitraum (Nenner) ermittelt. Zum Beispiel wurden in $200934 \mathrm{Pu}$ blikationen (13 aus 2007, 21 aus 2008) aus Gefässchirurgie zitiert. Bei insgesamt 94 als zitierfähig klassifizierten Publikationen (46 aus 2007, 48 aus 2008) ergab sich hierdurch ein JIF von 34/94=0,362.

Trotz vieler kritischer Stimmen gilt der JIF auch heute noch als einer der wichtigsten bibliometrischen Parameter zur Bestimmung der Qualität von Fachjournalen und - dieser Punkt wird besonders kontrovers diskutiert - zur Beurteilung einzelner Artikel und/oder des wissenschaftlichen Portfolios einzelner Forscher [11-14]. Die Beurteilung einzelner Fachartikel nur mit dem JIF ist jedoch irreführend, da auch in Zeitschriften mit hohem JIF - vereinfachend ausgedrückt - weniger gute bzw. wichtige Arbeiten erscheinen und - umgekehrt - in Journalen mit niedrigem JIF durchaus auch sehr beachtete Publikationen mit hohen Zita- tionszahlen vorliegen können. So zeigte eine Analyse in Nature, dass $89 \%$ des JIF des Jahres 2004 durch $25 \%$ der Artikel der Jahre 2002 und 2003 generiert worden waren [11]. Das bedeutet, dass $75 \%$ aller Artikel mit einem sehr hohen IF „belohnt" worden sind, obwohl sie nicht häufiger zitiert worden waren als andere $\mathrm{Pu}$ blikationen aus Zeitschriften mit niedrigerem JIF. Andere Autoren sprechen von der sog. 80/20 Regel, die besagt, dass $20 \%$ aller Artikel $80 \%$ der Zitationen erzielen und der JIF in keinem Fall repräsentativ für den „Impact“ einzelner Artikel sein kann [15]. Artikel eines Journals mit hohem JIF sind also nicht automatisch besser oder wichtiger als andere Artikel. Als Konsequenz des weit verbreiteten Missbrauchs des JIF sind Autoren allerdings bestrebt, ihre Originalarbeiten oder Review-Artikel in Zeitschriften mit hohem IF zu publizieren. Dies verstärkt die Kluft zwischen gut und weniger gut "gerankten" Zeitschriften.

\section{\) Der JIF gilt auch heute noch als einer der wichtigsten bibliometrischen Parameter zur Bestimmung der Qualität von Fachjournalen}

Als „zitierfähige“ Artikel eines Journals gelten zumeist Original- und Übersichtsarbeiten, CME-Artikel und weitere Artikel mit einem Umfang von mindestens drei Seiten, einem Abstract und einem aus mindestens 10 Zitaten bestehenden Literaturverzeichnis. Bei gleich bleibender Anzahl an Zitationen bedeutet dies, dass eine hohe Anzahl zitierfähiger Publikationen zu einem eher niedrigen JIF und eine niedrige Anzahl zitierfähiger Publikationen zu einem eher höheren JIF führt. Schriftleiter können also durch die Einwerbung sehr guter Review-Artikel (welche üblicherweise öfter zitiert werden) und die "Verknappung“ aller anderen zitierfähigen Artikel den JIF in begrenztem Umfang selbst steuern.

Darüber hinaus kann durch Eigenzitationen und die Aufforderung, Publikationen des eigenen Journals in anderen Zeitschriften zu zitieren, der JIF beeinflusst werden [16, 17]. Insbesondere das unkritische Zitieren von Arbeiten des eigenen Journals (Eigenzitate) ist allerdings nicht ganz ungefährlich, da es zur Aberkennung der Listung seitens des ISI führen kann. Aktuell wird eine Eigenzitationsrate von maximal $25 \%$ akzeptiert.

Weitere Kritikpunkte betreffen die niedrigeren JIF nicht englischsprachiger Zeitschriften [18], die IF-Überbewertung der Grundlagenforschung (im Vergleich zur klinischen Forschung [19]), die schlechte Vergleichbarkeit verschiedener Wissenschaftsdisziplinen und auch verschiedener Fächer innerhalb der Medizin (z. B. Neurologie versus Chirurgie) sowie den 2-Jahres-Zeitraum, der für die relevanten Zitationen herangezogen wird. Hierdurch könnten Fächer mit sehr hohem Innovationsgrad und entsprechend schnellen Publikationen begünstigt werden [20]. Dies bedeutet auch, dass Zitationen von Artikeln, deren Erscheinen länger als 2 Jahre zurückliegt, für den IF irrelevant sind. Hilfsweise wird deshalb gelegentlich ein 5-Jahres-JIF errechnet [8].

\section{Weitere Qualitätsindikatoren und Messparameter}

Aufgrund der o. g. Kritikpunkte wurden in den letzten Jahren eine Reihe weiterer bibliometrischer Messverfahren zur Beurteilung sowie zum Ranking wissenschaftlicher Fachzeitschriften vorgeschlagen. Hierzu gehören u. a.:

- der sog. Eigenfaktor, 
Tab. 2 Top-10-Artikel aus Gefässchirurgie mit den höchsten Zitationsraten (Artikel seit 1996)

\begin{tabular}{|c|c|c|c|}
\hline Autoren & Titel & $\begin{array}{l}\text { Jahr der } \\
\text { Publika- } \\
\text { tion }\end{array}$ & $\begin{array}{l}\text { Anzahl } \\
\text { Zitatio- } \\
\text { nen }\end{array}$ \\
\hline Hach W et al. & $\begin{array}{l}\text { Die krurale Fasziektomie zur Behandlung des inku- } \\
\text { rablen Gamaschenulkus (chronisches Faszienkom- } \\
\text { pressionssyndrom) }\end{array}$ & 1997 & 31 \\
\hline Noppeney T et al. & $\begin{array}{l}\text { Ergebnisse des Qualitätssicherungsprojektes Vari- } \\
\text { zenchirurgie der Deutschen Gesellschaft für Gefäß- } \\
\text { chirurgie }\end{array}$ & 2005 & 25 \\
\hline Torsello G et al. & $\begin{array}{l}\text { Projekt,Qualitatssicherung Karotischirurgie' der } \\
\text { Deutschen Gesellschaft für Gefäßchirurgie - Zwi- } \\
\text { schenauswertung nach } \\
7534 \text { Rekonstruktionen }\end{array}$ & 1997 & 21 \\
\hline Noppeney T et al. & $\begin{array}{l}\text { Leitlinie zur Diagnostik und Therapie des Krampf- } \\
\text { aderleidens der Deutschen Gesellschaft für Phlebo- } \\
\text { logie, der Deutschen } \\
\text { Gesellschaft für Gefäßchirurgie, des Berufsver- } \\
\text { bandes der Phlebologen e. V. und der Arbeitsge- } \\
\text { meinschaft der niedergelassenen Gefäßchirurgen } \\
\text { Deutschlands e. V. Entwicklungsstufe S2 }\end{array}$ & 2004 & 20 \\
\hline Hach W et al. & $\begin{array}{l}\text { Chirurgische und konservative Behandlung einer } \\
\text { transfaszial progredierenden Varikophlebitis der } \\
\text { Stammvenen und der Perforansvenen }\end{array}$ & 1996 & 20 \\
\hline Hach W et al. & $\begin{array}{l}\text { Das theoretische Verständnis der, Rezidivvarikose } \\
\text { nach „Operation“" }\end{array}$ & 1998 & 19 \\
\hline Hach W et al. & $\begin{array}{l}\text { Die Graduierung der chronischen venösen Insuffi- } \\
\text { zienz }\end{array}$ & 2000 & 16 \\
\hline Eckstein $\mathrm{HH}$ et al. & $\begin{array}{l}\text { Positionspapier der Deutschen Gesellschaft für Ge- } \\
\text { fäßchirurgie und Gefäßmedizin (DGG) Gesellschaft } \\
\text { für operative, } \\
\text { endovaskuläre und präventive Gefäßmedizin zur } \\
\text { Qualifikation bei der Durchführung endovaskulärer } \\
\text { Eingriffe }\end{array}$ & 2010 & 13 \\
\hline Dietze S et al. & $\begin{array}{l}\text { Die Claudicatio-Skala (CLAU-S), ein krankheitsspezi- } \\
\text { fischer Fragebogen zur Erfassung der Lebensquali- } \\
\text { tät von Patienten } \\
\text { mit Claudicatio intermittens: Entwicklung und } \\
\text { Validierung }\end{array}$ & 1997 & 13 \\
\hline Allenberg JR et al. & $\begin{array}{l}\text { Klassifikation des infrarenalen Aortenaneurysmas } \\
\text { (AAA): Endovaskuläre oder konventionelle Chirur- } \\
\text { gie? }\end{array}$ & 1996 & 13 \\
\hline
\end{tabular}

- die Ablehnungsquote eines Journals und

- der SCImago Journal Rank (SJR).

Der sog. Eigenfaktor korreliert sehr stark mit der Anzahl der Zitationen eines Journals, allerdings werden keine Eigenzitationen berücksichtigt. Er wird mittlerweile im Journal Citation Report des ISI geführt $[8,21]$. Die Ablehnungsquote eines Journals wurde ebenfalls als bibliometrischer Messparameter vorgeschlagen, der allerdings stark mit dem JIF korreliert [22]. Der SCImago Journal Rank (SJR) ist (im Gegensatz zum JIF) im Internet frei zugänglich. Er erfasst eine deutlich grö- ßere Anzahl von Journalen und beansprucht, die Qualität der einzelnen Zitationen zu berücksichtigen. Dies führt zu einem unterschiedlichen Ranking wissenschaftlicher Fachzeitschriften. Bei der kürzlich durchgeführten Analyse der TOP 100 Journals veränderte die Anwendung des SJR die Rangposition im Vergleich zum JIF um im Median 32 Rangpositionen. Immerhin blieben auch nach Verwendung des SJR ca. $50 \%$ aller JIF-TopJournale in der Liste der 100 am besten gerankten Journale [23]. Wie an allen Messparametern wird auch am SJR-Ranking Kritik geübt (insbesondere hinsichtlich des Ländervergleichs) [24].
Ranking einzelner Publikationen oder einzelner Wissenschaftler

Aufgrund der o. g. Limitationen des JIF wurden eine Reihe anderer Möglichkeiten vorgeschlagen, die Qualität und den "Impact“ einzelner Artikel bzw. des wissenschaftlichen Portfolios einzelner Forscher zu messen. Hierzu gehören die Analyse der individuellen Zitationen, die z. B. bei SCOPUS sowohl hinsichtlich einzelner Artikel, aber auch hinsichtlich einzelner Forscher durchgeführt werden kann. Die Evaluation und das Ranking einzelner Forscher erfolgt heute auch durch Anwendung des sog. Hirsch-Index (h-Index), der über eine mathematische Formel die Anzahl der Publikationen und der Zitationen ins Verhältnis setzt. Ein h-Faktor von 10 bedeutet z. B., dass 10 Publikationen des betreffenden Autors mindestens 10-mal zitiert worden sind. Es macht keinen Unterschied, ob 10 oder 100 Publikationen 10-mal zitiert worden sind. Der hFaktor steigt erst an, wenn mehr als $10 \mathrm{Pu}$ blikationen mehr als 10-mal zitiert worden sind. Auch der h-Faktor einzelner Forscher kann bei SCOPUS nachgelesen werden. Manche medizinische Fakultäten fordern bei der Neubesetzung von Ordinariaten mittlerweile einen h-Faktor von mindestens 20. Nachteilig ist, dass jüngere Forscher beim h-Faktor systematisch benachteiligt werden [25].

\section{》) Beim h-Faktor werden jüngere Forscher benachteiligt}

Ein noch größeres Potenzial versprechen sog. ",article-level metrics“ bzw. „ALM“ bzw. „altmetrics (alternative metrics)“ [26-29]. So werden z. B. beim „open access“ Portal PLoS (Public Libray of Science) einzelne Artikel kontinuierlich unter den folgenden Aspekten analysiert: „viewed, saved, discussed, recommended, cited“'. So konnte z. B. gezeigt werden, dass von 100.000 Aufrufen („viewed“) einzelner PLoS-Artikel, ca. $25 \%$ als PDF Dokumente heruntergeladen werden aber nur ca. $0,2 \%$ später auch zitiert werden. Die alleinige Fokussierung auf die Kategorie „cited“ könnte also zu einer systematischen Unterschätzung der Bedeutung einzelner Artikel führen. Es wurde daher vor- 


\begin{tabular}{|c|c|c|c|}
\hline Autoren & Titel & $\begin{array}{l}\text { Jahr der } \\
\text { Publika- } \\
\text { tion }\end{array}$ & $\begin{array}{l}\text { Anzahl Zita- } \\
\text { tionen }\end{array}$ \\
\hline Trenner M et al. & $\begin{array}{l}\text { Trends in Therapie und Outcome des nicht rup- } \\
\text { turierten abdominellen Aortenaneurysmas in } \\
\text { Deutschland zwischen } 1999 \text { und } 2010\end{array}$ & 2013 & 8 \\
\hline Ringleb P et al. & $\begin{array}{l}\text { S3-Leitlinie Carotisstenose, Kap. 7: Symptome } \\
\text { und Diagnostik von Carotisstenosen] }\end{array}$ & 2012 & 7 \\
\hline Kühnl $A$ et al. & Status quo der Gefäßchirurgie in Deutschland & 2013 & 6 \\
\hline Ringleb P et al. & $\begin{array}{l}\text { S3-Leitlinie Carotisstenose, Kap. 8.2: Wann und } \\
\text { zu welchem Zeitpunkt besteht die Indikation } \\
\text { zur Operation oder zur Intervention einer asym- } \\
\text { ptomatischen/symptomatischen Stenose (ein- } \\
\text { schließlich Notfallindikation), inkl. Subgruppen, } \\
\text { die eher von einer operativen, endovaskulären } \\
\text { oder konservativen Therapie profitieren? }\end{array}$ & 2012 & 6 \\
\hline Sander D et al. & $\begin{array}{l}\text { S3-Leitlinie extracranielle Carotisstenose: Kap. 6: } \\
\text { Epidemiologie }\end{array}$ & 2012 & 5 \\
\hline Wozniak G et al. & Standardisierte Wundtherapie & 2011 & 5 \\
\hline Ringleb P et al. & $\begin{array}{l}\text { S3-Leitlinie Extracranielle Carotisstenose: } \\
\text { Kap. 8.17: Welche Patienten sollten mit welcher } \\
\text { Medikation konservativ behandelt werden? }\end{array}$ & 2012 & 4 \\
\hline Rümenapf G et al. & $\begin{array}{l}\text { Neue Konzepte zur interdisziplinären Versor- } \\
\text { gung von Patienten mit neuroischämischem } \\
\text { diabetischem Fußsyndrom (DFS) }\end{array}$ & 2012 & 4 \\
\hline Clevert DA et al. & $\begin{array}{l}\text { Ultraschallgesteuerte EVAR-Interventionen und } \\
\text { Follow-up-Diagnostik mit der kontrastmittelge- } \\
\text { stützten Sonographie und der Bildfusion }\end{array}$ & 2011 & 4 \\
\hline NowakT et al. & $\begin{array}{l}\text { Perioperative Medikation bei arteriellen Opera- } \\
\text { tionen: Was ansetzen und was absetzen? }\end{array}$ & 2011 & 4 \\
\hline
\end{tabular}

Tab. 4 Nationale und internationale Rankings der Zeitschrift Gefässchirurgie (SCIMAGO 2013, [7])

\begin{tabular}{|ll}
\hline Kategorie & Rang \\
\hline $\begin{array}{l}\text { "Cardiology and Cardiovascu- } \\
\text { lar Medicine" }\end{array}$ & 247 von 333 \\
\hline "Cardiology + Cardiovascular & 14 von 18 \\
Medicine", GERMANY & \\
\hline "Surgery", international & 285 von 379 \\
\hline "Surgery", GERMANY & 28 von 39 \\
\hline
\end{tabular}

geschlagen, ,usage, downloads, and citations" gemeinsam zu analysieren [28]. Zur Analyse der Kategorie „discussed“ können darüber hinaus Blogs oder soziale Medien (Twitter, Facebook etc.) verwendet werden, die Kategorie „recommended" wird bei PLoS mithilfe einer kommerziell erhältlichen Software (F1000Prime) dargestellt und ausgewertet.

Das AWMF-Positionspapier zur Evaluation der medizinischen Forschungsleistung aus dem Jahr 2014 empfiehlt ebenfalls eine Veränderung des Systems der Bewer-
In eine ähnliche Richtung geht die San Francisco Declaration on Research Assessment (DORA) aus dem Mai 2013 [15], welches ebenfalls die Verwendung der bisherigen "Journal-Metrics" zur Evaluation wissenschaftlicher Forschungsleistungen ablehnt. Herausgeber von Zeitschriften werden aufgefordert, den JIF als alleiniges Kriterium zu verlassen und besser eine ganze Reihe verschiedener Parameter (Eigenfaktor, SCImago, h-Index, 5-JahrsJIF etc.) transparent darzustellen. Zudem werden Institutionen (wie z. B. Universitäten und medizinische Fakultäten) darauf hingewiesen, dass der wissenschaftliche Inhalt einer Publikation wichtiger ist als "publication metrics" des betreffenden Journals. Die Beurteilung wissenschaftlicher Leistungen sollte $u$. a auch die Bedeutung für die tägliche Praxis berücksichtigen.

\section{Open-access- (OA-)Journale}

In den vergangenen 20 Jahren wurden zahlreiche Open-access- (OA-)Journale neu gegründet bzw. bestehende Zeitschriften, wie z. B. das British Medical Journal (BMJ) im Jahr 1998, vollständig online frei verfügbar gemacht. Mittlerweile verfügen die großen Verlage über eigene OA-Plattformen, wie z. B. BioMedCentral (Springer Verlag) oder die Public Library of Science (PloS). Vorteile der OA-Publikationsform sind $u$. a. die schnelle Bearbeitung von der Einreichung bis zur Publikation. Autorenrechte an der Publikation müssen in aller Regel nicht abgetreten werden. Die kostenlose Zugriffsmöglichkeit durch Leser und/oder Betroffene fördert zudem die weltweite Sichtbarkeit.

Prinzipiell stehen zwei Wege von Open-access- (OA-)Publikationen/Journalen zur Verfügung. Die alleinige Online-Publikation und Archivierung im Internet (gold $O A$ ) oder die Publikation einer Arbeit als Print-Version sowie die zusätzliche Archivierung in einem Online-Archiv (green $O A$ ) [31]. Letztere Option würde z. B. der in Heft 7/2014 vorgeschlagenen Weiterentwicklung der Gefässchirurgie in ein sog. Hybrid-Journal entsprechen [1]. Eine Analyse von ca. 17.000 in SCOPUS gelisteten Journalen zeigte, dass medizinische und medizinnahe 
Journale folgendermaßen eingeteilt werden können [32]:

- ca. $10 \%$ in die Kategorie gold $O A$,

- 30-40\% in die Kategorie green OA und

- $>50 \%$ in die Kategorie non-OA.

In dieser Analyse konnte auch gezeigt werden, dass Green-OA-Journale mit einem Wert von 1,6 etwa doppelt so häufig zitiert wurden wie Gold-OA-Journale oder die Non-OA-Journale (jeweils ca. 0,8 Zitationen). Auch erzielten die GreenOA-Journale einen deutlich höheren SJR bei SCImago. Diese Analyse belegt, dass die Kombination aus Print-Journal und OA-Journal (bzw. OA-Publikationen) zu einer deutlich höheren Sichtbarkeit führen kann als die alleinige OA- oder die Non-OA-Publikation.

OA-Publikationen werden aber auch durchaus kritisch betrachtet. Dies betrifft z. B. das gelegentlich intransparente Review-Verfahren [33] und die Frage danach, ob OA-Publikationen auch nach längeren Zeitintervallen noch abrufbar sein werden. Vereinzelt wird auch befürchtet, dass Autoren ihre Arbeiten ohne jedes Peer-Review-Verfahren (freeto-publish and free-to-read) ins Internet stellen könnten [34]. Der größte Kritikpunkt sind allerdings die sog. article-processing charges (APC), die sich bei den großen Verlagen um ca. 3000 US-Dollar oder 2,200 $€$ bewegen (Elsevier, Springer etc.) und auch bei reinen Online-Journalen immer noch 500-1,000 € betragen [35]. Diese Kosten werden erhoben, um einerseits den Ausfall der Einnahmen aus Abonnements zu kompensieren, andererseits sind dies aber auch willkommene zusätzliche Einnahmen [36]. Typischerweise sind die APC bei Journalen, die keinen JIF haben und nicht in SCOPUS gelistet werden, niedriger, als bei Journalen, die im Journal Citation Report gelistet sind und über einen hohen JIF verfügen [37]. Letztendlich müssen Autoren oder Institutionen die APC tragen, um „open access“ publizieren zu können. Da hohe APC auch abschrecken können, versuchen eine zunehmende Anzahl von Zeitschriften, ihre Arbeiten zumindest teilweise „open access" verfügbar zu machen, ohne APC zu erheben $[3,4,38,39]$. Dies ist z. B. durch externe Unterstützung durch z. B. Fachge- sellschaften, hohe Werbeeinnahmen oder andere Business-Modelle möglich [40].

\section{Perspektiven für die Zeitschrift Gefässchirurgie}

Nach Ansicht der Autoren werden Leser und Leserinnen aus klinisch sehr aktiven Fächern auf absehbare Zeit nicht auf ein Print-Journal verzichten wollen. Dies hat auch etwas damit zu tun, dass Chirurg(inn)en ihre Arbeitszeit weniger am Schreibtisch, als im OP, auf der Station oder der Ambulanz verbringen. Darüber hinaus zeigen die o. g. Analysen sehr deutlich, dass unsere Zeitschrift nicht nur beim JIF, sondern auch bei anderen Indikatoren (SJR, Eigenfaktor etc.) in nationalen und internationalen Rankings zu weit hinten liegt.

Es muss im Interesse der Zeitschrift und ebenso im Interesse der gesamten deutschsprachigen Gefäßchirurgie sein, national und international wesentlich stärker wahrgenommen (und damit auch zitierfähig) zu werden. Dieser Weg kann sinnvollerweise nur über englischsprachige (ggf. aus dem Deutschen übersetzte) Artikel erfolgen, die „open access“ (sog. Hybrid-Journal oder „green open access,) verfügbar sind. Eine konsequente Umsetzung dieses Vorschlags würde dem eigenen akademischen Nachwuchs helfen, der deutschsprachigen Gefäßchirurgie ein weltweites Echo ermöglichen und der Zeitschrift Gefässchirurgie im Konzert der vaskulären und chirurgischen Journale eine echte Perspektive bieten. Darüber hinaus wäre ein Qualitätsschub eingereichter Manuskripte zu erwarten, da gute Arbeiten mit einer Open-access-Verfügbarkeit belohnt werden könnten. Insgesamt erscheint dieser Weg daher sehr erstrebenswert.

Die Kosten für diese Weiterentwicklung müssen - wohl oder übel - von den drei Fachgesellschaften übernommen werden. Aus Sicht der Autoren müsste daher zukünftig wieder ein größerer Teil der Mitgliedsbeiträge für die Zeitschrift Verwendung finden, um den Mitgliedern von DGG, SGG und ÖGG ein inhaltlich und konzeptionell hochwertiges Journal zu bieten! Auch die Deutsche Gesellschaft für Angiologie hat es gemeinsam mit ihren Partnern aus Österreich und der
Schweiz geschafft, mit VASA ein international wahrgenommenes Journal zu etablieren (JIF von 1,4 in 2013). Zur Erinnerung: in den 90er Jahren musste investiert werden, um die Zeitschrift zum Laufen zu bringen. Aufgrund steigender Mitgliederzahlen sind die Kosten in den folgenden Jahren deutlich gefallen und machen heute nur noch ca. ein Drittel der damaligen Kosten aus, und dies für 8 Hefte (in den 90er Jahren 4 Hefte) und nach Umsetzung einer Reihe inhaltlicher Verbesserungen.

Aus Sicht der Autoren sollte die Gefässchirurgie daher - trotz aller Kritik und Bedenken - die möglichst rasche Wiedererlangung einer Listung beim ISI und dem damit verbundenen JIF anstreben. Unabhängig vom Journal-Ranking sollte Gefässchirurgie ihren Autoren die entsprechenden "article-level metrics (AML)“ noch besser darstellen. Immerhin ist die Anzahl der Downloads über die Jahre ständig angestiegen. Der „Impact“ mancher Artikel aus Gefässchirurgie könnte also wesentlich stärker sein als es die eher niedrigen Zitationsraten vermuten lassen.

Zusammenfassend eröffnen sich für die Gefässchirurgie sehr positive Perspektiven. Diese können aber nur realisiert werden, wenn DGG, ÖGG und SGG den Stellenwert ihres „Organs“ neu definieren und entsprechende intellektuelle und finanzielle Ressourcen aufzubringen bereit sind.

\section{Korrespondenzadresse}

Univ.-Prof. Dr. H.-H. Eckstein

Klinik und Poliklinik für Vaskuläre und

Endovaskuläre Chirurgie

Klinikum rechts der Isar, Technische Universität

München, Ismaninger Straße 22, 81675

München

hheckstein@web.de

\section{Einhaltung ethischer Richtlinien}

Interessenkonflikt. H.-H. Eckstein, H. Söllner und A Kühnl geben an, dass es kein Interessenkonflikt besteht.

Dieser Beitrag beinhaltet keine Studien an Menschen oder Tieren.

\section{Literatur}

1. Eckstein H-H (2014) Die Zukunft der Zeitschrift Gefässchirurgie. Gefässchirurgie 19(7):611-613 
2. http://www.aerzteblatt.de/int, Deutsches Ärzteblatt International [01.05.2015]

3. http://www.aerzteblatt.de/down.asp?id=4167, Deutsches Ärzteblatt - Ärzte-Verlag. Sechs Gründe, im Deutschen Ärzteblatt zu publizieren [11.05.2015]

4. http://onlinelibrary.wiley.com/journal/10.1111/ \%28ISSN\%291610-0387/homepage/ProductInformation.html, Deutsche Dermatologische Gesellschaft. JDDG: Journal der Deutschen Dermatologischen Gesellschaft [11.05.2015]

5. http://pb.rcpsych.org, The British Journal of Psychiatry - Bulletin [01.05.2015]

6. http://www.scopus.com, Scopus [01.05.2015]

7. http://www.scimagojr.com, SCImago. SJR - SClmago Journal \& Country Rank. 2007 [03.05.2015]

8. http://thomsonreuters.com/en/products-services/scholarly-scientific-research/research-management-and-evaluation/journal-citation-reports.html, Reuters T. Journal Citation Reports [03.05.2015]

9. Garfield E (1972) Citation analysis as a tool in journal evaluation. Science 178(4060):471-479

10. http://www.thomsonreuters.com/about-us/company-history/, Institute for Scientific Information (ISI). [09.05.2015]

11. Campbell $P$ (2008) Escape from the impact factor. Ethics Sci Environ Polit 8:5-7

12. Nallamothu BK, Luscher TF (2012) Moving from impact to influence: measurement and the changing role of medical journals. Eur Heart J 33(23):2892-2896

13. Winit-Watjana W (2014) Impact fators: misuse and initiatives. Arch Pharma Pract 5:3-5

14. Seglen PO (1997) Why the impact factor of journals should not be used for evaluating research. BMJ 314(7079):498-502

15. Bladek M (2014) DORA San Francisco declaration on research assessment. Coll Res Libr News 75(4):191-196

16. Agrawal AA (2005) Corruption of journal impact factors. Trends Ecol Evol 20(4):157

17. Fassoulaki A, Paraskeva A, Papilas K, Karabinis G (2000) Self-citations in six anaesthesia journals and their significance in determining the impact factor. Br J Anaesth 84(2):266-269

18. Vinther S, Rosenberg J (2012) Impact factor trends for general medical journals: non-English-language journals are lacking behind. Swiss Med Wkly 142:w13572

19. Eck NJ van, Waltman L, Raan AF van, Klautz RJ, Peul WC (2013) Citation analysis may severely underestimate the impact of clinical research as compared to basic research. PLoS One 8(4):e62395

20. Fröhlich G (2003) Gegen-Evaluation: Der ImpactFaktor auf dem Prüfstand der Wissenschaftsforschung. BUKO INFO, S 1-4. http://www.iwp.jku. at/Froehlich/docs/ImpactFaktorPr\%C3\%BCfstand. pdf. Zugegriffen: 10 Mai 2015

21. Fersht $A$ (2009) The most influential journals: impact factor and eigenfactor. Proc Natl Acad Sci USA 106(17):6883-6884

22. Fang FC, Casadevall A (2011) Retracted science and the retraction index. Infect Immun 79(10):3855-3859

23. Falagas ME, Kouranos VD, Arencibia-Jorge R, Karageorgopoulos DE (2008) Comparison of SCImago journal rank indicator with journal impact factor. FASEB J 22(8):2623-2628

24. Mañana-Rodríguez J (2014) A critical review of SCImago Journal \& Country Rank. Res Eval doi: 10.1093/reseval/rvu008
25. Hirsch JE (2005) An index to quantify an individual's scientific research output. Proc Natl Acad Sci USA 102(46):16569-16572

26. Dinsmore A, Allen L, Dolby K (2014) Alternative perspectives on impact: the potential of ALMs and altmetrics to inform funders about research impact. PLoS Biol 12(11):e1002003

27. Fenner M (2013) What can article-level metrics do for you? PLoS Biol 11(10):e1001687

28. Lin J, Fenner M (2013) Altmetrics in evolution: defining and redefining the ontology of article-level metrics. Inf Stand Q 25(2):1-8

29. Neylon C, Wu S (2009) Article-level metrics and the evolution of scientific impact. PLoS Biol 7(11):e1000242

30. Herrmann-Lingen C, Brunner E, Hildenbrand S, Loew TH, Raupach T, Spies C et al (2014) Evaluation of medical research performance - position paper of the Association of the Scientific Medical Societies in Germany (AWMF). Ger Med Sci. doi:10.3205/000196

31. Björk B-C, Laakso M, Welling P, Paetau P (2014) Anatomy of green open access. J Assoc Inf Sci Technol 65(2):237-250

32. Miguel S, Chinchilla-Rodriguez Z, Moya-Anegon F de (2011) Open access and scopus: a new approach to scientific visibility from the standpoint of access. J Am Soc Inf Sci Technol 62(6):11301145

33. Bowman JD (2014) Predatory publishing, questionable peer review, and fraudulent conferences. Am J Pharm Educ 78(10):176

34. Wakeford R (2013) Open access publication - always a good thing? J Radiol Prot 33(2):E9-E11

35. Tzarnas S, Tzarnas CD (2015) Publish or perish, and pay - the new paradigm of open-access journals. J Surg Educ 72(2):283-285

36. Moustafa K (2015) Commentary: open access, open business, closed fairness! Account Res 22(4):245-247

37. Solomon DJ, Björk B-C (2012) A study of open access journals using article processing charges. J Am Soc Inf Sci Technol 63(8):1485-1495

38. Abbasi K (2009) Open access for the JRSM. JR Soc Med 99(3):101

39. http://jrs.sagepub.com/, Royal Society of Medicine. Journal of the Royal Society of Medicine [11.05.2015]

40. Pimm J (2014) Open access publishing - a quiet revolution. Psychiatr Bull (2014) 38(1):1-2

\section{Hier steht eine Anzeige.}

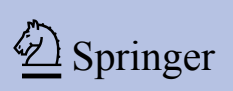

